THURSDAY, DECEMBER I, I898.

\section{THE SHIVERING EARTH.}

Seismology. By John Milne, F.R.S., F.G.S. Pp. xvi +320 . (London: Kegan Paul, Trench, Trübner, and Co., Ltd.)

PROFESSOR MILNE spent more than twenty years in Japan. When he went there he was experienced as a miner and geologist ; and had spent years in Newfoundland, Iceland, Arabia, Siberia and Mongolia as an explorer. He was Professor of mining and geology, and later, of seismology, but for twenty-three years he seems to have devoted himself to the one subject, seismology. The Transactions of the Seismological Society of Japan are mainly of his writing. He has published books and numerous papers in England. Through his influence the Japanese Government has established many seismological observatories. He seems to have made a thorough trial of hundreds of seismometers. He enlisted the observational services of many of the foreigners living in Japan. As Secretary of the British Association Committee he has written numerous valuable reports. $\mathrm{He}$ has induced all the engineers and architects in Japan to build in accordance with the conclusions drawn by him from his observations. During the last two years he has been the means of establishing twenty-three seismological stations over the world, and he considers it his duty to keep in communication with them all.

In all these years he seems to have missed no chance of supplementing his own mathematical and scientific knowledge by that of anybody whom he could induce to study his subject. He has a pleasant style, and knows from experience as a popular lecturer exactly how his subject may be made interesting to the general reader, and the result is a very readable book which probably contains all that is worth knowing on this subject at the present period of its development. Twenty such volumes might have been written if the author had cared to touch on all the crude speculation of quasi-scientific workers which has from time to time been published.

The earth, about whose interior we know as much as Carlyle's ephemera knew about the lunar theory, is intensely hot under enormous pressure at all depths below a few miles. As the inside cools it contracts, and the crust must also get smaller, and so we have all sorts of crumpling and buckling actions going on slowly always; gradual changes of slope which show themselves mainly by changes of sea level. A small vertical fall or rise in water level may accompany enormously great changes in land area. A lift of all the Tertiary mountainous districts to an average height of 4000 feet would seem to have required only a vertical fall of the water level of twenty-six feet, although the actual breadth of land exposed at coasts may possibly have been very great; and by the possible repeated exposure and submergence of great areas of sedimentary beds, accompanying the probable discontinuous rise of the mountains. Prof. Milne throws an unexpected light on the well-known fact that the great mountain-forming epochs in geological history, epochs of great brady-seismical and volcanic activity, have a close chronological identity with the periods NO. I 5 I 8, VOL. 59] of coal formation. That there has been crumpling in the past is evident enough; but it is interesting to note how we are getting evidence that it is still going on ; that the surface of the earth almost everywhere is changing in slope, and falling and rising. Not only have we tilting due to mere change of atmospheric pressure; to rain being better retained by some kinds of crops than others ; to the attraction of the moon ; to the melting of polar ice ; to the erosion of land by sea and river ; and the more or less continuous slipping of soil on steep slopes, but to a far more important extent locally, by the deposition of sediment at the mouths of rivers. Every now and again the bending crust undergoes fracture, especially at the bases of monoclines. Some kinds of rock keep fracturing continually; others yield without fracture for long times, and when they fracture they do it with violence; faults are suddenly formed, possibly at great depths, and the motion, the sudden shiver or vibration is transmitted to every part of the earth. If the fault occurs near the surface of the earth, other evidence of it appears than the temporary passage of an imperceptible or terrifying shiver ; in minor dislocations, horizontal displacement and sometimes actual contraction. Thus in Japan in the Neo valley in I89I some plots of ground were diminished 30 per cent. in breadth; river beds were permanently narrowed; forests slipped down from mountain sides to block up valleys and form great lakes ; the whole land on one side of the valley lowered in level, and a boundary ridge of high mountains with it. Any one who looks at maps of the world published by the Electric Cable Companies will notice how, along the west side of South America, and at many places elsewhere, a cable is not brought along the coast directly from one place to another; for safety it goes out to sea a great distance. If we did not have actual evidence of it, we could not believe in the numerous sudden large changes of sea bottom which occur, fracturing submarine cables. Except in Japan no people are so enthusiastic in the establishment of Prof. Milne's observatories as those interested in such cables. In I 888 the simultaneous fractures of three submarine cables between Australia and Java by an earthquake caused Australia to mobilise its naval and military forces; and on several occasions Prof. Milne has been able to answer questions of the Colonial Government as to the cause of fracture of cables.

The earthquake or shiver is transmitted to all parts of the earth. The student of acoustics may imagine how the complicated system of vibration gets reflected and refracted and changed in character as it travels, and how different the complete record must be at a place near to the primary disturbance from what it is six thousand miles away, and how anywhere it depends on the local character of the ground. And yet, in spite of this complication, there are interesting general rules which have been derived by the author from observation. A disturbance which has travelled 6000 miles, is recorded by preliminary simple tremors which may have periods of 5 to $\mathrm{I}_{2}$ seconds, the more decided movements having periods of from 20 to 40 seconds. At places nearer, the motion is more complicated; the preliminary tremors have periods of from ${ }^{\circ} 04$ to $\cdot 2 \mathrm{sec}^{\circ}$; the decided movements, say with ranges of motion to to $20 \mathrm{~mm}$., have 
periorls of 2 to $2 \frac{1}{2} \mathrm{sec}$; movements of $\mathrm{I}$ to $\mathrm{I} \cdot 5 \mathrm{~mm}$. range, which constitute the greater part of many records, have periods of about $I$ sec. The record ends in simple vibrations of periods $I \cdot 5$ to 4 seconds, like waves of the ocean after a storm.

It is to be presumed that magnetic and other messages are constantly passing to us through the earth, which, if we could only read them-if we knew anything about the cause of terrestrial magnetism, for example-would tell us something about the inside of our habitation. Earth shivers which have travelled from Japan to England seem to be the only messages coming to us through the earth's body which we are able to understand, and which can give us at present any knowledge of what that body is like.

Vibrations from gunpowder explosions in the earth are found to travel faster if the explosions are more violent. Mallet's experiments gave velocities of from 250 to 500 metres per sec. The velocities deduced from the HellGate explosion vary from 1300 metres per second to 6200 . More sensitive apparatus records higher velocities, because it has been acted upon by the smaller tremors, and these travel faster than others. Prof. Milne gives a list of earthquake speeds actually observed.

Counting time from the first record anywhere and distance from the place of this first record, measuring either by an arc on the earth's surface, or by a chord, we may look upon the following speeds as not unusual.

\begin{tabular}{c|c|c|c}
\hline \multicolumn{2}{c|}{ Distance from origin. } & Apparent velocity in kiloms. per sec. \\
\hline In degrees. & In kiloms. & Along arc. & Along chord. \\
\hline & & & \\
20 & 2,200 & 2 to 3 & 2 to 3 \\
50 & 5,500 & 5 & 5 \\
80 & 8,800 & 8 & 7.5 \\
100 & II, 100 & 10 & 8.8 \\
120 & 13,200 & $12 ?$ & $10 ?$ \\
160 & 17,700 & $16 ?$ & $105 ?$ \\
& & $16 ?$ \\
\hline
\end{tabular}

The more decided state of motion which arrives with more or less of definiteness of transition after the tremors, travels at a much slower rate ( 1 to $3 \frac{1}{2}$ kiloms. per sec.), and hence the time of duration of the preliminary tremors tells us the distance to the origin. Hence when a seismograph record is made in the Isle of Wight, the distance through which the motion has travelled may generally be estimated with some accuracy, and hence also the violence of the initial disturbance may be guessed at.

It seems to be out of the question that the preliminary tremors have travelled on the earth's surface either as Lord Rayleigh's surface waves or as distortional waves or condensational waves. We can only understand their propagation if we imagine the stuff in the earth at great depths as having cubic elasticity very much greater than that of the best steel, but it is not so easy to understand how such enormous elasticity can be possessed by it. Those students of nature who assume that the behaviour of rock on the earth's surface enables them to speak with easy certainty as to the behaviour of rock at enormous pressure and temperature, must surely find some difficulty in understanding the above observations, just as there are celestial phenomena which must surely disturb the equanimity of the unimaginative persons who apply their farthing rush-light laboratory experience without modification or reserve to all the phenomena of the universe.

The author describes the instruments used now to note or accurately record or measure the motion of the ground or of any point in a structure when an earthquake or shiver passes. The invention of a good instrument for each kind of motion has exhausted much mathematical and inventive genius. It will be found that Prof. Milne is wonderfully fair to other inventors. There seems still to be some doubt as to how much of the motion recorded as horizontal motion of the ground is perhaps something different, an effect of tilting of the instrument. The study of the whole subject has undoubtedly added to our knowledge of vibration phenomena, and I should think that this book will be found of great value by young engineers in charge of electric light stations, where the most important problem of the present time is how to protect citizens from the annoying vibration of reciprocating steam engines. At some future period it may enter the minds of manufacturers of engines that the lineal descendant of Newcomen's slow reciprocating pumping engine is not perhaps the best kind of quick speed motor to use in a thickly inhabited district, and that although passengers by steamer must put up with such a nuisance because there is no forbidding law, the manufacturers of electric energy may easily be compelled to reform their methods of working. The study of all such vibration can be carried on only by the use of instruments such as Prof. Milne describes They have already given useful information in showing how wasteful of coal a badly balanced locomotive may be ; in picking out badly constructed parts of the permanent way in railways, in detecting loose parts in bridge construction, and, more important than any of these, they are now giving us information as to the dangerous vibrations of some bridges when trains pass over.

One important result that seems to be gradually establishing itself in the minds of observers is that the number of shakes $s$ per day (vigorous enough for record on a particular kind of instrument) at any place at the time $t$ days after a large earthquake, and at the distance $n$ miles from the epicentre, is a not very. complex sort of function of $n$ and $t$. From the numbers given by Prof. Milne, I venture to say that the law for the after-shocks of the 189 I earthquake is something like

$$
s=\mathrm{II} 7 \div\left(e^{t / 3 \mathrm{~T}+n / 27}\right)
$$

an expression that is worth some study as representing fairly well the result of observation. An enormous amount of labour has been given to the search for a periodicity in earthquakes. It does seem that seismic and barometric maxima coincide, and possibly because of this, or because of snow accumulation, there is greater seismic activity in winter than in summer, although more destructive earthquakes seem to occur in summer. The much looked for dependence of seismic activity on the moon's position is not yet established so well as the No. I 5 I 8 , VOL. 59] 
annual and semi-annual periodicities; nor has an effect of the ocean tides yet shown itself. It does not seem to be established that there is any connection between earthquakes and electric or magnetic or auroral phenomena. Slow changes of slope of the earth's surface at any place are continually going on ; these are more pronounced in the direction of the dip of certain strata. Those that are mostly of importance in astronomical observatories are due to differences in soils and crops in retaining power for moisture; and Prof. Milne seems to have made a careful study of soils, not merely in regard to their absorption and retention of moisture from the air, but also in regard to their condensation of vapour coming up from beneath, and the results of his observations and experiments seem as if they might be of value to agriculturists.

The author dwells at some length upon curious earth pulsations and earth tremors which seem to be unconnected with earthquakes. They occur everywhere, and their study ought to be of importance to all who have an interest in astronomical or magnetic observatories or in exact measurement of any kind; for exansple, in careful weighing.

Earth pulsations have periodic times of two to three minutes, or even ten or more minutes, beginning and ending for no known reason, lasting for one to three hours. Are they connected with the curious sea-swells of the Pacific which recur annually at very high tide and last for twenty-four hours? Then there are the curious earth tremors, not to be confounded with the effects of traffic on roads-they are storms lasting eight to twelve hours, sometimes two to three days, never less than three hours.

No doubt in some cases mere air currents inside the covers of the instruments produce some of the effects observed, but in most cases they are real earth tremors, due possibly to expansions and contractions of the soil by heat and other causes, but this will not explain everything; nor will meteorological changes; nor will winds acting upon the ground in the neighbourhood. The level and slope of ground change perpetually, and the changes seem never to be quite continuous. Prof. Milne cites many observations which show the great importance of the study of these earth tremors in connection with changes of barometric pressure and the escape of fire-damp in mines.

I know that what I have here jotted down after reading this most interesting and valuable scientific work will give only a very poor idea of its contents, and the author will consider that his views are described very crudely. But what can be done in a short notice of such a book? Every sentence in the book contains the result of much thought and observation, and yet it is a book which is just as easy to read as the report of a popular lecture. One has also the feeling that the writer is appealing for sympathy and co-operation of all kinds, without which his great work in the establishment of observatories cannot go $\mathrm{c}_{11}$; it is the kind of appeal that one reads between the lines of a traveller's book sometimes, an appeal that the author does not know that he is making. It certainly adds to the interest of an already interesting subject.

JoHN PERRY.

\section{COFFEE AND INDIA-RUBBER IN MEXICO.}

Coffee and India-rubber Culture in Mexico. Preceded by Geographical and Statistical Notes on Mexico. By Matias Romero. Pp. xxvi +417 . (New York and London: G. P. Putnam's Sons, The Knickerbocker Press, 1898.)

A $\mathrm{T}$ a time when both the products mentioned at the A head of the title-page of this book are attracting a great amount of attention as important cultural industries suitable for many of the British Colonies, as well as for other parts of the world, and when india-rubber or caoutchouc especially continues to increase in value and demand, anything bearing on the cultivation of these plants is sure to be eagerly sought after.

It would almost seem that in selecting the title for his book Mr. Romero had in view the probability of catching readers by reversing the order of its correct title, which should more properly stand as "Geographical and Statistical Notes on Mexico: followed by Chapters on Coffee and India-rubber Culture," for in a volume of 417 pages it is not till we arrive at p. $28 \mathrm{I}$ that the consideration of the cultivation of coffee is commenced, and it is finished at p. 359. Again, with india-rubber this subject is disposed of in the thirty-three concluding pages of the book. The statistical portion of the book, therefore, occupies its greatest bulk, and is placed first in order. Besides which Mr. Romero candidly says in his introduction that the papers on coffee and rubber were written about a quarter of a century ago, and simply appear now as a translation without any attempt at bringing them up to date; while the geographical and statistical notes were only just published when the introduction was written in January last.

It may be of some interest, as showing how the book has been put together, to quote a few paragraphs from the author's introduction. At p. v. he says, speaking of the article on coffee :

"I published in Mexico three editions of my manual, correcting and adding to each new one, the last one being published in July I 874 . There was at the time no interest in coffee culture, and very little attention was therefore paid to my manual. By the advice of a friend, I placed in a book-store about fifty copies on sale, and four or six years later only two or three had been sold."

Again, on p. vi. Mr. Romero gives his reasons for not bringing his matter up to modern times as follows:

"I am very sorry that my present engagements have prevented me from revising this paper up to date; that is, changing, such views expressed in the same as my experience has taught me not to be entirely correct, at least, in so far as other regions outside of the southern coast of Chiapas are concerned, as that would require more time than I can afford; and, in my inability to do that work, I prefer to use the paper I wrote long ago exactly in the shape in which it then came out. Since that time all circumstances and conditions of coffee raising have materially changed."

In the introduction to the paper on rubber culture Mr. Romero puts forward the same reasons for not revising his paper. which he says :

"I publish now exactly as it came out over a quarter of a century ago." 